Supporting Information for:

\title{
Efficient negative photochromism by the photoinduced migration of photochromic merocyanine/spiropyran in the solid-state
}

Tetsuo Yamaguchi, ${ }^{1}$ Kamonnart Jaa Imwiset ${ }^{2}$ and Makoto Ogawa ${ }^{1^{*}}$

${ }^{1}$ School of Energy Science and Engineering and ${ }^{2}$ School of Molecular Science and Engneering, Vidyasirimedhi Institute of Science and Technology (VISTEC), 555 Moo 1 Payupnai, Wangchan, Rayong 21210, Thailand

E-mail: makoto.ogawa@vistec.ac.th.

1. Small angle X-ray diffraction

2. Thermogravimetric analysis (TGA)

3. $\mathrm{N}_{2}$ adsorption/desorption isotherm of MPSS

4. UV-vis absorption spectra of SP toluene solution before and after photoinduced adsorption

5. UV-vis absorption spectra of the negative photochromic hybrids

6. SEM images 


\section{Small angle $X$-ray diffraction}

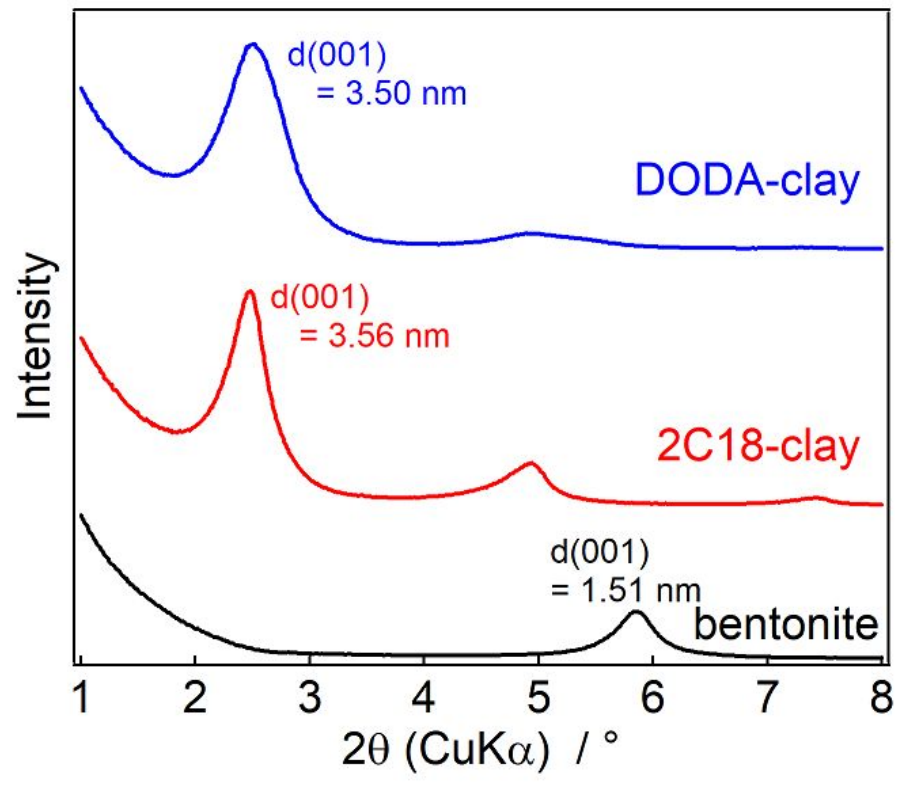

Fig. S1 X-Ray powder diffraction patterns of the bentonite (black line), 2C18-clay (red line) and DODA-clay (blue line). 
2. Thermogravimetric analysis (TGA)

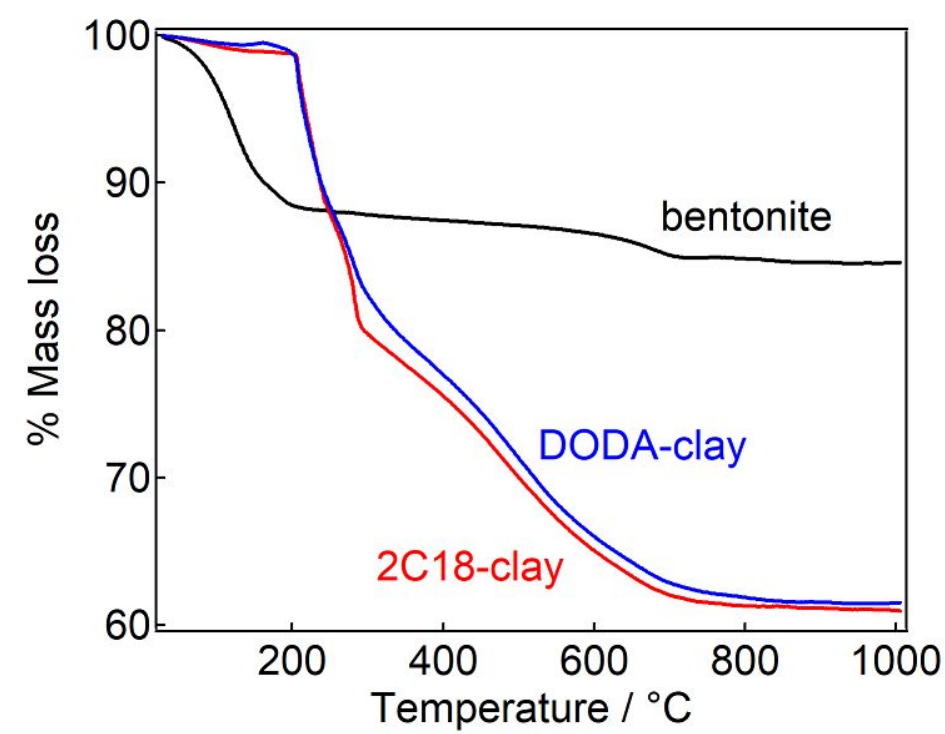

Fig. S2 Thermogravimetric analyses of the bentonite, 2C18-clay, and DODA-clay with the heating rate of $10^{\circ} \mathrm{C} / \mathrm{min}$.

\section{3. $\mathbf{N}_{2}$ gas adsorption}

(a)

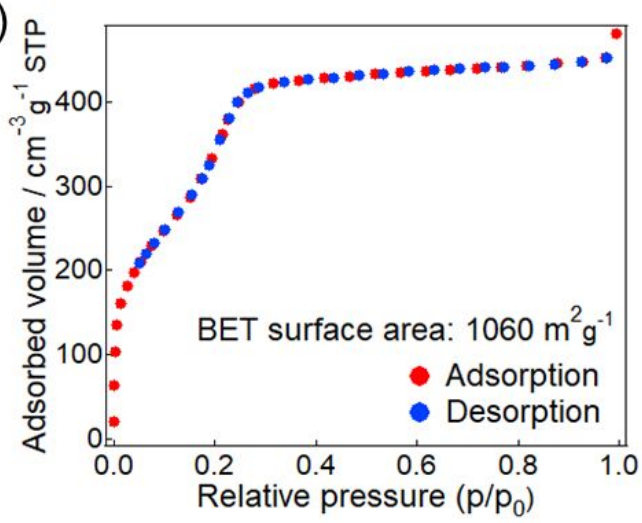

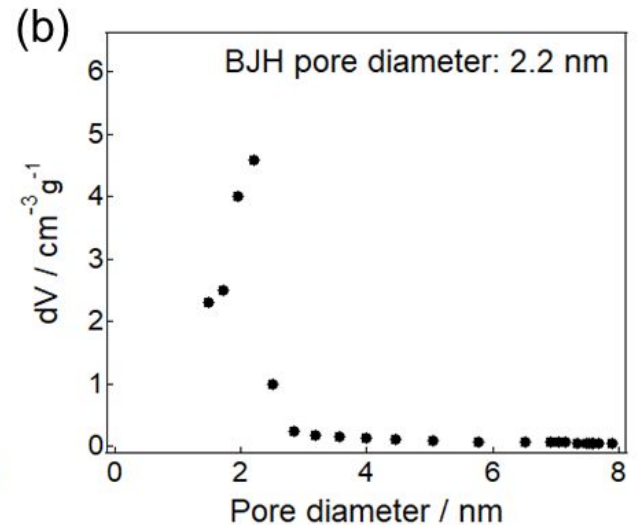

Fig. S3 (a) $\mathrm{N}_{2}$ adsorption-desorption isotherms and (b) BJH pore distribution for mesoporous silica sphere. 
4. UV-vis absorption spectra of SP toluene solution before and after photoinduced adsorption

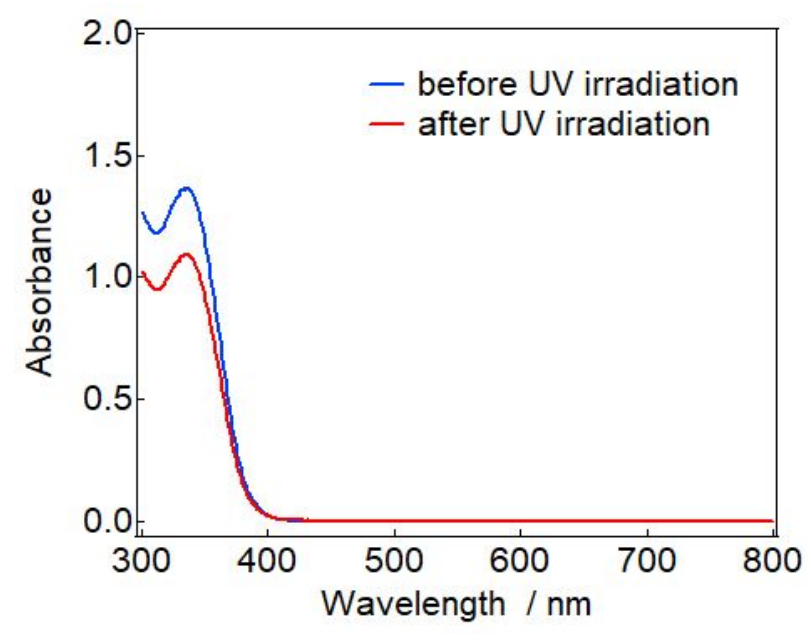

Fig. S4 UV-vis absorption spectra of SP suspensions of MPSS before and after the UV irradiation in the optical cuvette with $2 \mathrm{~mm}$ light path length. 


\section{UV-vis absorption spectra of the negative photochromic hybrids}

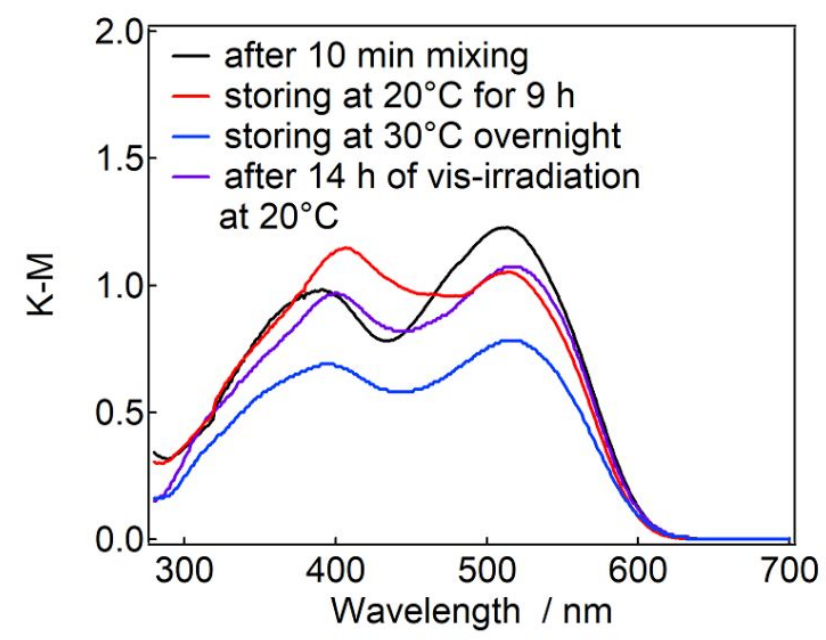

Fig. S5 UV-vis absorption spectra of 2C18-clay/MPSS(MC) after 10 min mechanical mixing (black line), after storage for $9 \mathrm{~h}$ in the dark at $20^{\circ} \mathrm{C}$ in the dark (red line), after storage overnight at $30^{\circ} \mathrm{C}$ in the dark (blue line), after visible light irradiation and subsequent storage for $14 \mathrm{~h}$ at $20^{\circ} \mathrm{C}$ in the dark (violet line).

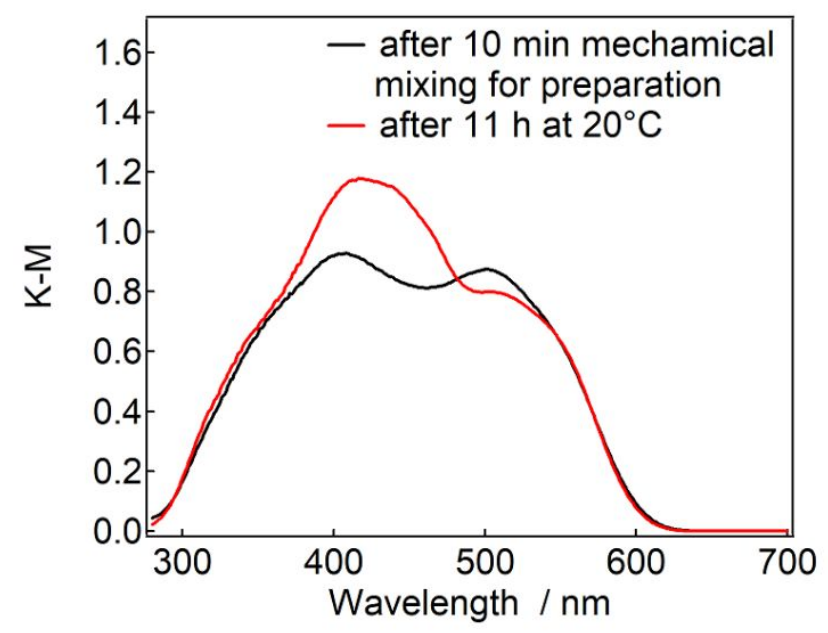

Fig. S6 UV-vis absorption spectra of DODA-clay/MPSS(MC) after 10 min mechanical mixing for preparation and after $11 \mathrm{~h}$ storage in the dark at $20^{\circ} \mathrm{C}$.

DODA-clay containing SP (designated as DODA-clay(SP)) had absorption at 348 and $542 \mathrm{~nm}$ and a shoulder at $415 \mathrm{~nm}$ (Fig. S1). The absorbance at $400 \mathrm{~nm}$ appeared and that at 542 $\mathrm{nm}$ increased by the UV irradiation for $2 \mathrm{~min}$, suggesting that DODA-clay(SP) showed similar photochromism to 2C18-clay(SP). 


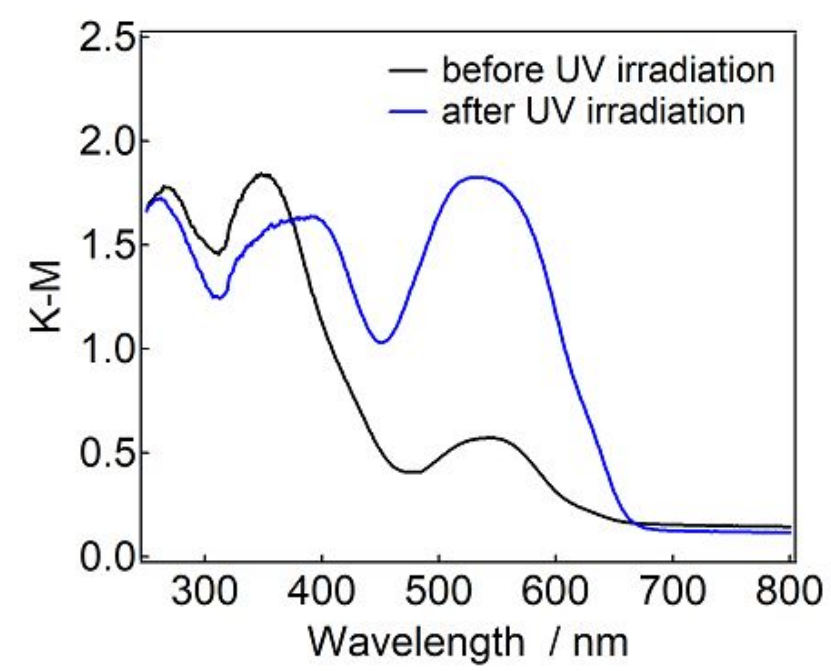

Fig. S7 Absorption spectra of DODA-clay(MC) before and after UV irradiation.

Time profiles at $510 \mathrm{~nm}$ and $545 \mathrm{~nm}$ after $2 \mathrm{~min}$ UV irradiation are shown in Fig. S6, which corresponded to the change from blue line to red line in Fig. $5 \mathrm{~b}$ in the main manuscript. The decrease of the absorption continued in $400 \mathrm{~min}$ and followed singe exponential equation (eq. 1 in the main manuscript) with a half-life of $1.0 \mathrm{~h}$.

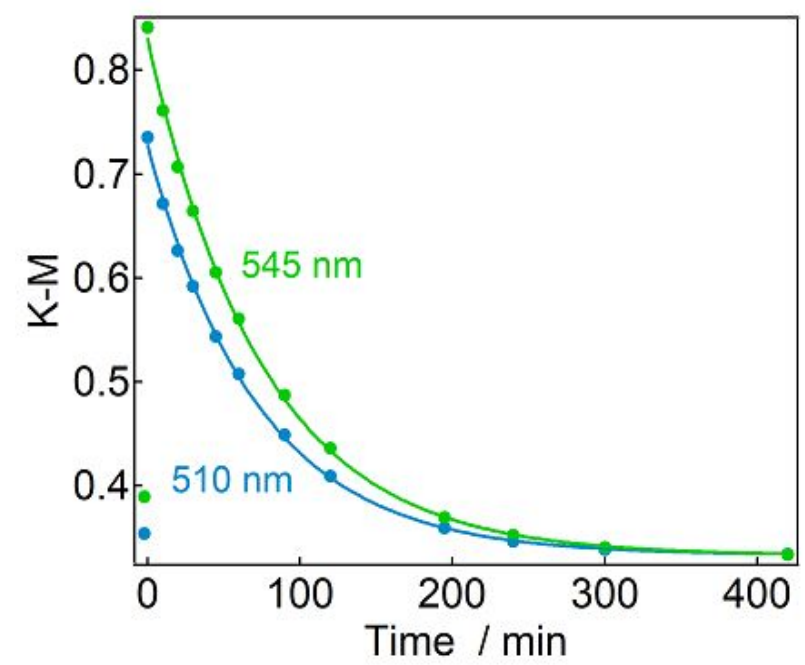

Fig. S8 Time profiles at $510 \mathrm{~nm}$ and $545 \mathrm{~nm}$ after $2 \mathrm{~min}$ UV irradiation. 


\section{SEM images}
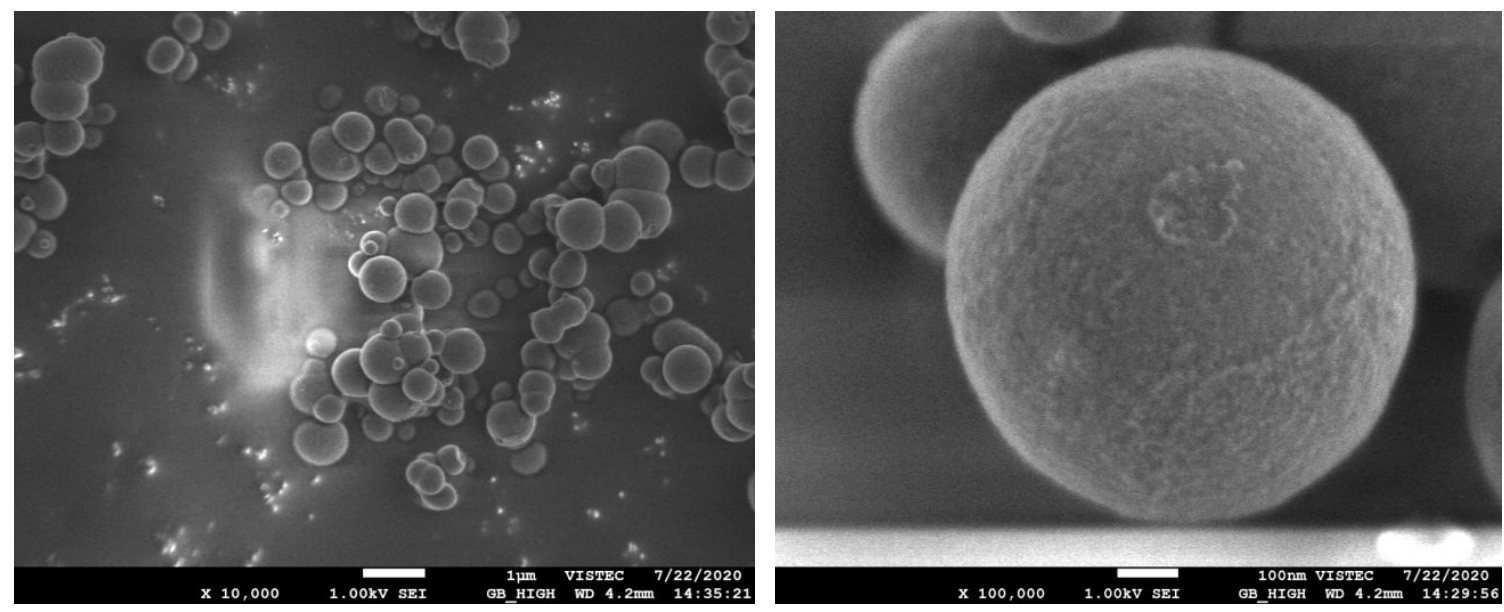

Fig. S9 SEM images of MPSS.
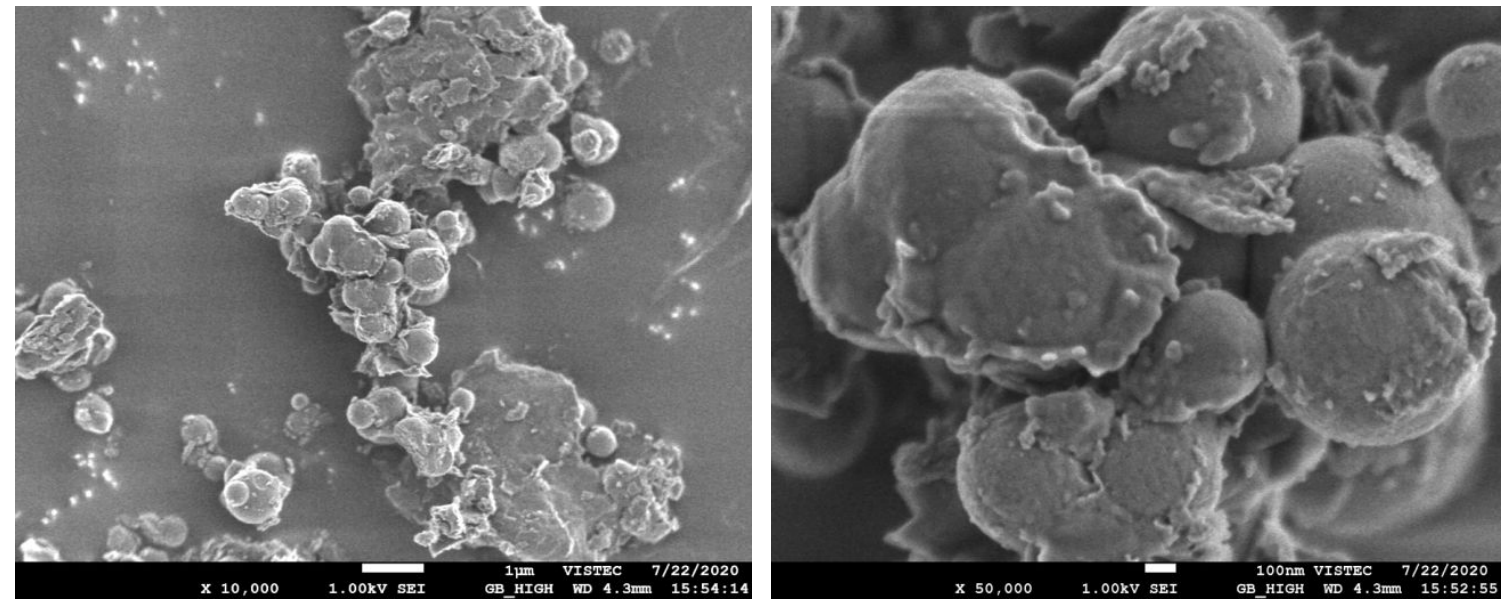

Fig. S10 SEM images of 2C18-clay/MPSS(MC)
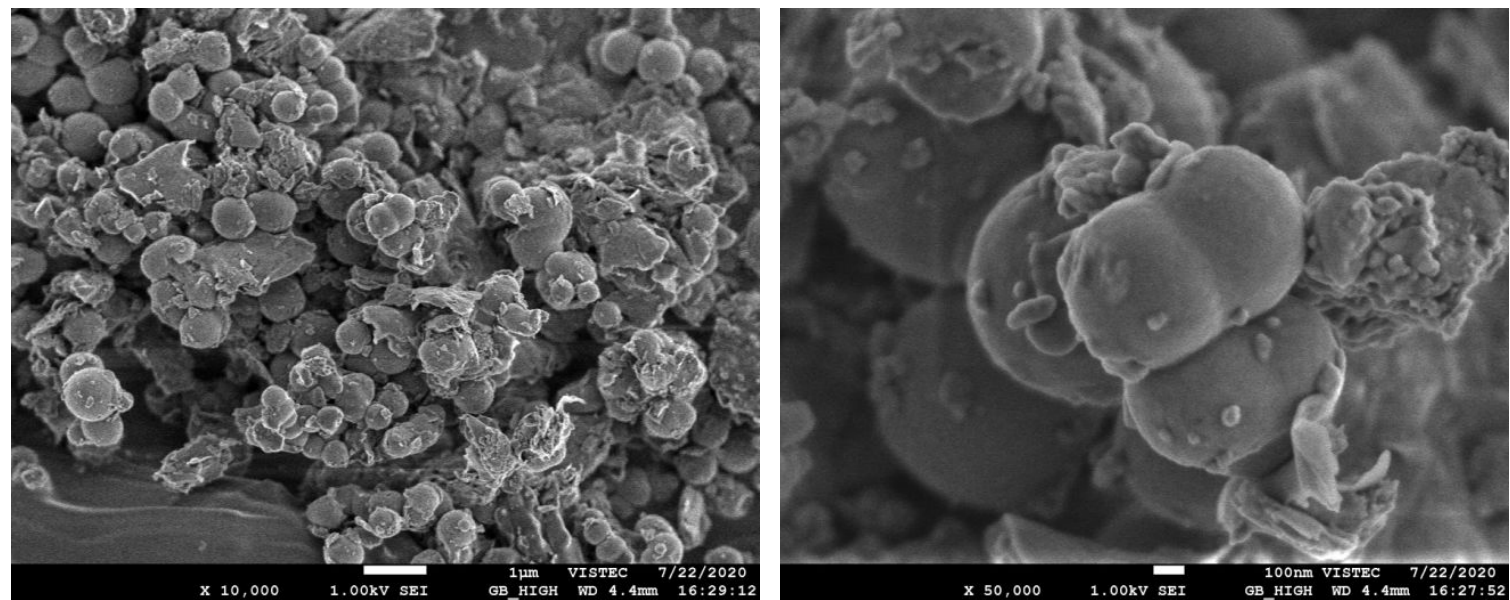

Fig. S11 SEM images of DODA-clay/MPSS(MC) 